\title{
Intelligent data aggregation inspired paradigm and approaches in IoT applications
}

\author{
Xiaohui Yuan ${ }^{\mathrm{a}}$ and Mohamed Elhoseny ${ }^{\mathrm{b}, *}$ \\ ${ }^{a}$ Department of Computer Science and Engineering, University of North Texas, Denton, Texas, USA \\ ${ }^{\mathrm{b}}$ Faculty of Computer and Information Sciences, Mansoura University, Egypt
}

\begin{abstract}
Intelligent data aggregation approaches and technologies play an important and increasing role in practice due to the widespread adoption of mobile devices in many applications. Motivated not only by the increasing number of mobile devices, but also their ever-growing computing and sensing capabilities, there have been efforts to leverage these devices as destination for offloading computations/data in the context of IoT applications. The special issue aims at presenting a collection of high-quality research papers on the state-of-the-art in the smart data aggregation and routing techniques.
\end{abstract}

Keywords: Smart network applications, scientific computing and data management, data-driven service management, spatial data techniques, data processing in smart city applications

Intelligent data aggregation approaches and technologies play an important and increasing role in practice due to the widespread adoption of mobile devices in many applications. In this scenarios Internet of things (IoT) will be dominated by large wireless devices like smart sensor and smartphone and smart wearable devices. This requires technologies to handle this massive data traffic with high mobility. The considerable amount of data generated by these devices needs efficient handling and security for diverse applications.

Motivated not only by the increasing number of mobile devices but also their ever-growing computing and sensing capabilities, there have been efforts to leverage these devices as a destination for offloading computations/data in the context of IoT applications. Such a trend has also been referred to as dew computing in the literature. However, current research in the area is still focused on augmenting mobile clients

\footnotetext{
${ }^{*}$ Corresponding author. Assistant Professor, Faculty of Computer and Information Sciences, Mansoura University, Egypt. E-mail: Mohamed_elhoseny@mans.edu.eg.
}

via fixed computing resources (e.g., local servers and computer clusters), so substantial unexploited computing and sensing capabilities remain "at the edge." Therefore, many research opportunities to exploit mobile devices in the context of intelligent data aggregation for IoT applications arise.

The special issue aims at presenting a collection of high-quality research papers on the state-of-the-art in the smart data aggregation and routing techniques. We are soliciting original contributions in both theoretical studies and state-of-the-art practical applications. We through the call for paper through some conference and research community, we collected more than 300 papers. Based on the peerreview comments, we carefully selected 50 papers for this special issue. All these 50 papers can be divided into five categories which include into data mining, deep learning, cloud computing, intelligent algorithm, and IoT technologies \& application.

There are eleven papers are focused on data mining technologies and the algorithms for big data. To improve the optimization of the education system, 
article [1] use the data mining algorithm to classify and summarize the education data and improve the accuracy of teaching content. An optimization and updating scheme is put forward from the algorithm flow and evaluation model, and an adequate evaluation model is established by effectively evaluating the developing state of wisdom education. The experimental results show that the proposed method can improve the teaching efficiency, and the proposed method also can make the teaching content can be sent to the particular students according to its interest. Article [4] applied the TOP-K algorithm into a competency model of innovation and entrepreneurship team, which can archive $20 \%$ performance improvement than the traditional method. Article [6] proposed a prefix span algorithm for intrusion detection, which can up to $2 X$ speedup than the mostly used start-of-art methods. Article [12] uses data mining algorithms to analyze the effectiveness of ideological and political education. Article [9] proposed a new heuristic decision tree algorithm and then studied the urban green space ecological network by using the proposed algorithm. To better mine, the required data information quickly based on condition matching, an optimized Apriori and FP-Growth association rule mining algorithm is proposed in the article [40]. Article [36] analyzed the application of computer technology in trajectory prediction of moving objects in the data mining algorithm and proposes a trajectory analysis method based on the structure and cloud computing motion capture algorithm. Banking systemic risk contagion is similar to the spread of infectious disease. Article [37] analyzed the of banking systemic risk contagion by introduced the network dynamic time-variant contagion kinetics model, which archived a significant system performance improvement. Article [42] surveys the development data of some Chinese enterprises, and the author concludes some interesting points based on the analyzing of these data by using the fuzzy logic and SEM model. While article [44] get some other conclusion by analysis the macroeconomics background, meanwhile, article [16] analyzed the forestry industry in China, which is another interesting view. Inspired by the theory of complex network link prediction and the topic model, article [46] proposed an acquisition method for users' browsing behavior preference. Focused on the combined technical actions in hip-hop movements, article [48] introduced the fuzzy analysis method for bio-mechanical industrial action, which concludes some interesting results. While arti- cle [49] propose to build the flow shop model with full-loaded constraints and maximum wagons within the stage, article [50] discussed the impact of corporate innovation on stock price crash risk and the moderating effect of investors' attention on the relationship between the two under the triple effect of enhancing confidence, interpreting information and releasing panic.

Deep learning is an essential role in artificial intelligent techniques; there are nine papers are focused on deep learning technologies and applications. To solve the problem of carrier-based aircraft model which caused by the nonlinearity and uncertainty, article [6] proposed an adaptive sliding mode control based on dynamic recurrent fuzzy neural network and then applied the proposed model in the longitudinal landing system. To ensure the optimization of numerical processing, article [8] proposed a numerical simulation based deep learning algorithm which can improve the gas explosion efficiency in confined space. In the article [12], the authors design a human-computer interactive English experience teaching based on fuzzy set and BP neural network. In the article [14], the authors proposed a model that combines multiple deep CNN features and use fusion strategies to explore multi-scale features relationship. The model shows a good way of tailoring pre-trained CNN models to fine-grained ship classification, which have lower cost in computation and storage compared with some state-of-the-art CNN methods and also achieves the important classification performances in FGVC-Aircraft and Stanford Cars datasets. Article [39] propose a novel ant-colony based clustering algorithm, which can efficiently select the most valuable data points for the next step of learning. Article [43] use a genetic algorithm based optimization method to improve the parameters of BP neural network, which can archive a good performance improvement of BP neural network, and then the author applied the improved method into the nonlinear model of dynamic coal blending. Article [45] proposed a BP neural networkbased optimization method for poverty alleviation fund audit mode. Article [3] studied the parameterization of safety behavior coefficient in the process of security management based on the cloud computing model.

A cloud aggregator is a type of cloud broker that packages and integrates multiple cloud computing services into one or more composite services. This integration, which includes services from various 
cloud providers, is typically more cost-effective to the customer than purchasing each service separately. A cloud aggregator, in turn, may benefit from charging a premium for providing more than a straightforward resale agreement. There are five papers focused on cloud integration and data aggregation algorithms. We introduce these five articles together.

The intelligent algorithm is another important aspect of artificial smart technology and big data analysis. There are ten articles focused on artificial intelligent and big data analysis. Because of all these ten papers are relevant to the intelligent algorithm, so we introduce all these papers together. Article [5] established a new integrated English teaching network platform based on an artificial intelligence method. To promote the rapid application of new technology of driverless vehicles, article [41] considers the influence of people, vehicles, roads, and traffic management on driverless vehicles, and constructs a highway safety evaluation model based on support vector machine. Article [28] proposed a new intelligent algorithm to optimize the investment model under stochastic interest rate and stochastic volatility. In the article [30] the author makes economic benefit analysis of green building based on fuzzy logic and bilateral game model. By introducing such factors as economic benefits, cognition and government policies, the author constructs an evolutionary game model, which provides a basis for improving the economic benefits of green buildings. In the article [32], the authors propose an underwater object detection method, which uses enhanced spectral residual (SR) saliency detection and fuzzy segmentation. The authors adopt a two-phase mechanism, which divides visual object detection into detecting saliency map and image segmentation to obtain "proto object." Due to the continuous improvement of industrial production requirements and green manufacturing demand, manufacturing enterprises and factories need to optimize and improve the system structure continually. Article [33] analyzes the reason of the coal blending error in coal blending process and the optimization scheme of intelligent control of coal blending process and then optimized the overall structure of the coal blending system, improved the coal blending system which uses the fuzzy control system. Article [34] proposed a novel fingerprint feature extraction method for the communication radiation source. Article [47] studied the separation performance of aircraft background acoustic blindness and then introduced a new separation model.
The Internet of Things (IoT) promises to reshape entire industries. The business value can be profound-ranging from digitizing an organization's internal operations and customer experience to unlocking disruptive new digital products and business models. There are fifteen papers focused on IoT and its application technologies. The establishment of physical education courses in colleges plays a decisive role in promoting the personal development of students. However, as the teaching time of the physical education courses is limited, it has become an urgent problem to improve the students' professional skills in the limited time of teaching. How to solve the problem that Fuzzy measure can't be fused. Article [7] studied the collaborative innovation model algorithm for artificial intelligence. To further explore the state monitoring of rotating machinery, article [10] proposed a new updating scheme to improve the rotating performance. Article [11] expounds the development history of the bilingual context in English and Chines, then the author proposed a new algorithm and applied the proposed algorithm to optimize and update the Bilingual flow. Article [24] focused on how to solve the problem that the fuzzy measure cannot be fused, and then proposed the optimization and updating scheme from the algorithm flow and evaluation model. To meet the demand of the number of at least AP positioning in the positioning area, article [31] introduced the improved fuzzy $\mathrm{C}$ clustering algorithm and support vector machine location of interference source location and area, which can archive a good system performance than the state-of-the-art work. The conventional JSEG method is not very accurate for the target edge localization in segmentation results. To solve this problem, article [35] proposed an improved segmentation method of remotely sensed image based on JSEG algorithm and fuzzy c-means (FCM) with spatial constraints. Specific to the trajectory planning of intelligent electric vehicles with multiconstraints in some unknown environment, using rolling optimization principle of predictive model control for reference, article [38] proposed an online trajectory planning algorithm based on a rolling window.

In conclusion, this special issue would not have been possible without the help of many people. As guest editors, we would like to take this opportunity to thank the authors for their contributions and the reviewers for their invaluable comments and timely responses. We also would like to thank the JIFS 
Editor-in-Chief and staff for their support during the preparation and production of this special issue.

\section{References}

[1] S.G. Wang, Smart Data Mining Algorithm for Intelligent Education, Journal of Intelligent \& Fuzzy Systems (JIFS)

[2] E.E. Mahmoud and B.H. ALharthi, Secure communications via modified complex phase synchronization of two hyperchaotic complex models with identical linear structure and adjusting in nonlinear terms, Journal of Intelligent \& Fuzzy Systems (JIFS)

[3] S.C. Tian, K. Tang, P.F. Yang, A.F. Jia and Melvin, Hailey. Secure Cloud Computing Model for Communication Network Management, Journal of Intelligent \& Fuzzy Systems (JIFS)

[4] L. Zhao and Z.R. Tian, Noura Metawa. Data Mining-based Competency Model of Innovation and Entrepreneurship, Journal of Intelligent \& Fuzzy Systems (JIFS)

[5] L. Zhao, L.J. Chen, Q. Liu, M.Y. Zhang and Henry Copland. Artificial Intelligence-based Platform for Online Teaching Management Systems, Journal of Intelligent \& Fuzzy Systems (JIFS)

[6] Q.D. Zhu and Z.B. Yang. Dynamic recurrent fuzzy neural network-based adaptive sliding control for longitudinal automatic carrier landing system, Journal of Intelligent \& Fuzzy Systems (JIFS)

[7] Song X. Intelligent System for Products Personalization and Design Using Genetic Algorithm, Journal of Intelligent \& Fuzzy Systems (JIFS)

[8] X.W. Li, H. Chen and Bryan Ariann. Computer Network Security Evaluation Model Based on Neural Network, Journal of Intelligent \& Fuzzy Systems (JIFS)

[9] B.S. Sun, J.P. Qian, K.Y. Qu and Draper Geoffrey M. Heuristic Decision Tree Model for Ecological Urban Green Space Network Construction, Journal of Intelligent \& Fuzzy Systems (JIFS)

[10] Liu H. X, Condition Monitoring and Fault Diagnosis of Rotating Machinery Based on Feature Extraction and Expression of Vibration Signals, Journal of Intelligent \& Fuzzy Systems (JIFS)

[11] X.M. Yang, Intelligent Construction of English-Chinese Bilingual Context Model based on CBR, Journal of Intelligent \& Fuzzy Systems (JIFS)

[12] K. Kong, J.B. Li and Y.M. Wang. Human-computer interactive teaching model based on fuzzy set and BP neural network, Journal of Intelligent \& Fuzzy Systems (JIFS)

[13] M.A. Mabrok and A. Abdel-Aty, Pattern detection for time series trajectories in human in the loop applications, Journal of Intelligent \& Fuzzy Systems (JIFS)

[14] S.Z. Huang, H.S. Xu and Z.X. Xia, Multi-feature fusion of convolutional neural networks for Fine-Grained ship, Journal of Intelligent \& Fuzzy Systems (JIFS)

[15] H. Peng, L. Liu, J.Y. Liu, Lewis and Johnwb R. Network Traffic Anomaly Detection Algorithm Using Mahout Classifier, Journal of Intelligent \& Fuzzy Systems (JIFS)

[16] S. Chen and X.M. Zhang. Optimization of regional forestry industrial structure and economic benefit based on deviation share and multi-level fuzzy comprehensive evaluation, Journal of Intelligent \& Fuzzy Systems (JIFS)
[17] A.M. Shehata, Model predictive control of electric power and reserve dynamic dispatch including demand response, Journal of Intelligent \& Fuzzy Systems (JIFS)

[18] X.J. Wang, Research on human resource performance and decision-making evaluation based on fuzzy mathematics and clustering model, Journal of Intelligent \& Fuzzy Systems (JIFS)

[19] Liu Ta, Han Dongping and R.K. Arvind Shriram, Intuitionistic Fuzzy Measures of Enterprise Eco-efficiency and Its Influencing Factors, Journal of Intelligent \& Fuzzy Systems (JIFS)

[20] X.X. Ouyang and Y.Q. Dai. Supply chain equilibrium and transportation network efficiency based on MSA algorithms and multilayer decision model, Journal of Intelligent \& Fuzzy Systems (JIFS)

[21] S.N. Mohanty, J.R. Parvinb, K.V. Kumar, K.C. Ramya, S.S. Rani and SK. Lakshmanaprabu, Optimal Rough Fuzzy Clustering for User Profile Ontology based Web Page Recommendation Analysis, Journal of Intelligent \& Fuzzy Systems (JIFS)

[22] R. Mahesh and T. Meyyappan, Fuzzy based Cell Generalization to Improve the Data Utility with Minimal Loss of Information, Journal of Intelligent \& Fuzzy Systems (JIFS)

[23] A. Ahmad, B. Whitworth, F. Zeshan, L. Janczewski, M. Ali, M.H. Chaudary and R. Friedman, A relation-aware multiparty access control, Journal of Intelligent \& Fuzzy Systems (JIFS)

[24] L. Zhao, Y. Liu, L.J. Chen, J.C. Zhang and Koomey Jonathan G. English Oral Evaluation Algorithm Based on Fuzzy Measure and Speech Recognition Technology, Journal of Intelligent \& Fuzzy Systems (JIFS)

[25] X. Zhou, Dynamic Monitoring and Management System for Land Resource based on Parallel Network Algorithm and Remote Sensing, Journal of Intelligent \& Fuzzy Systems (JIFS)

[26] X.L. Song, J. Guo, Y. Wu, D.G. Yang, J.H. Wang and B. Tao, A New Model for Quorum Sensing and Image Simulation of Plant Rhizosphere Microorganisms, Journal of Intelligent \& Fuzzy Systems (JIFS)

[27] Tang Xiaogangr, W. Sun'an, L. Mingxue, L. Litian and K. Shankar, Channel Usability Pattern Guided Spectrum Prediction and Sensing, Journal of Intelligent \& Fuzzy Systems (JIFS)

[28] T. Luo and Noura Metawa, Intelligent Algorithm of Optimal Investment Model under Stochastic Interest Rate and Stochastic Volatility, Journal of Intelligent \& Fuzzy Systems (JIFS)

[29] P.U. Maheswari, P. Manickam, K.S. Kumar, A. Maseleno and S. Kathiresan, Bat optimization algorithm with Fuzzy based PIT Sharing (BF-PIT) Algorithm for Named Data Networking (NDN), Journal of Intelligent \& Fuzzy Systems (JIFS)

[30] Z.L. Chen, Economic benefit analysis of green building based on fuzzy logic and bilateral game model, Journal of Intelligent \& Fuzzy Systems (JIFS)

[31] Y.F. Chen, T.H. Du, S.G. Sun and C.D. Jiang, Indoor location of interference source with fuzzy $\mathrm{C}$ clustering cruising AP based on double fingerprint database, Journal of Intelligent \& Fuzzy Systems (JIFS)

[32] H. Feng, X.H. Yin, L.Z. Xu, G.F. Lv, Q. Li and L.L. Wang, Underwater salient object detection jointly using improved spectral residual and Fuzzy c-Means, Journal of Intelligent \& Fuzzy Systems (JIFS) 
[33] Q. Yin, J.B. Hu, H.M. Tong, S.Y. Song and G.Q. Zhang. Optimization of coal blending process control based on fuzzy self-tuning

[34] K. Li, J.Y. Zhang, Y.K. Lei and Cyn Ra, A Novel Fingerprint Feature Extraction Method for Communication Radiation Source, Journal of Intelligent \& Fuzzy Systems (JIFS)

[35] J. Shen, H. Chen, M.X. Xu, C. Wang and H. Liu, Intelligent Image Segmentation Model for Remote Sensing Applications, Journal of Intelligent \& Fuzzy Systems (JIFS)

[36] L.Z. Liu, F.G. Liu and Byron Ky, Data Mining-based Model for Motion Target Trajectory Prediction, Journal of Intelligent \& Fuzzy Systems (JIFS)

[37] Y.H. Gu, S.Z. Zhu, Z.P. Yang and Y.J. Zhao, Research on banking systemic risk contagion based on network dynamic time-variant contagion kinetics model, Journal of Intelligent \& Fuzzy Systems (JIFS)

[38] P.C. Sheng, J.G. Ma, D.P. Wang, W.Y. Wang and M. Elhoseny, Intelligent Trajectory Planning Model for Electric Vehicle in Unknown Environment, Journal of Intelligent \& Fuzzy Systems (JIFS)

[39] H. Huang, H.J. Deng, Y.Q. Sheng and X.Z. Ye, Accelerating convolutional neural network-based malware traffic detection through ant-colony clustering, Journal of Intelligent \& Fuzzy Systems (JIFS)

[40] X.D. Yang, X.X. Lin, X.L. Lin and Q. Woric, Application of Apriori and FP-Growth Algorithms in Soft Examination Data Analysis, Journal of Intelligent \& Fuzzy Systems (JIFS)

[41] Wang G, Safety Evaluation Model for Smart Driverless Car Using Support Vector Machine. Journal of Intelligent \& Fuzzy Systems (JIFS)

[42] X.Y. Tian and P.K. Nie, Econometric analysis of political connection affect corporate credit financing constraints based on fuzzy logic and SEM model. Journal of Intelligent \& Fuzzy Systems (JIFS)
[43] R. Wang, S.C. Yang and D.X. Wang, Intelligent Piezoelectric Peristaltic Linear Driving Model Based on Neural Network, Journal of Intelligent \& Fuzzy Systems (JIFS)

[44] Y. Wang and H.F. Wang, Quantitative analysis of regional economic indicators prediction based on grey relevance degree and fuzzy mathematical model, Journal of Intelligent \& Fuzzy Systems (JIFS)

[45] H.L. Wang and Kamrul Islam, An Optimization Model for Poverty Alleviation Fund Audit Mode Based on BP Neural Network, Journal of Intelligent \& Fuzzy Systems (JIFS)

[46] X. Liu, Y.J. Zhou and Z.G. Wang, Acquisition method of users' browsing behavior preference based on the fusion of social network link and theme model, Journal of Intelligent \& Fuzzy Systems (JIFS)

[47] L.Z. Jia, D.L. Cheng, C.J. Yi and Sandra Zick, The Separation of Aircraft Background Acoustic Blindness Based on EEMD-ICA, Journal of Intelligent \& Fuzzy Systems (JIFS)

[48] K.Y. Han, X.P. Liu, S.K. Ping and P. Wang, Bio-mechanical fuzzy analysis on the technical action of windmill coupling swipe in hip-hop movement, Journal of Intelligent \& Fuzzy Systems (JIFS)

[49] Y. Jing, S.Y. Guo, Z.H. Zhang and J. Ebrim, Model and algorithm of wagon-flow allocating in marshalling station based on sequence theory, Journal of Intelligent \& Fuzzy Systems (JIFS)

[50] J. Hou, J.F. Meng and L.M. Zhu, Empirical analysis of corporate innovation, investor focus and stock slumps risk based on fuzzy mathematics and function optimization, Journal of Intelligent \& Fuzzy Systems (JIFS) 